\title{
Zeitkontext und Buchmarkt
}

\section{Der württembergische Buchdruck in der frühen Reformationszeit}

\section{Maßhalten und Geschäftstüchtigkeit kann man} gerade mit Blick auf den Buchdruck in den ersten Jahrzehnten des 16. Jahrhunderts als charakteristische Eigenschaften der württembergischen Bevölkerung erkennen. Jedenfalls traf für Württemberg nur bedingt zu, was der päpstliche Nuntius Hieronymus Aleander (1480-1542) in einer Depesche vom Wormser Reichstag am 8. Februar 1521 nach Rom berichtete: "Täglich regnet es lutherische Schriften in deutscher und lateinischer Sprache ... Es wird hier gar nichts Anderes verkauft als Schriften Luthers, und selbst am kaiserlichen Hofe, denn die Leute halten ganz erstaunlich zusammen und haben Geld in Menge".

Im Territorialstaat Württemberg waren die Rahmenbedingungen und Voraussetzungen komplexer als in anderen Regionen Deutschlands. Württembergische Städte zählten nicht zu den besonders produktiven Druckzentren. Mit der 1477 gegründe-

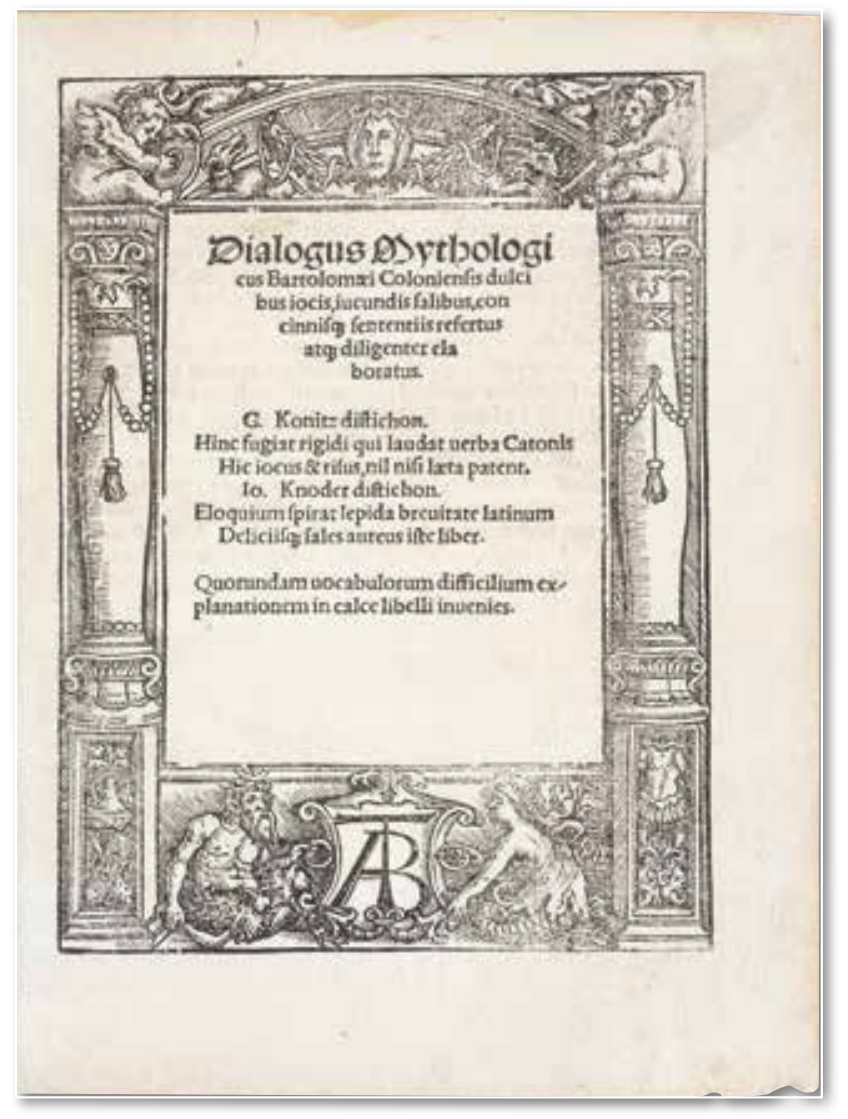

Abb. 1: Melanchthons vorreformatorische Zeit (HBF 1570) ten Universität Tübingen bestand ein institutionell fassbarer Kreis von Autoren und Leserschaft für wissenschaftliche Literatur. Daher verwundert es, dass selbst hier zunächst nur von 1498 bis 1501 , von 1511 bis 1516 und dann erst wieder ab 1523 gedruckt wurde. Tübingen war in dieser Frühphase eine temporäre Durchgangsstation für Drucker, die mehrmals im Lauf ihres Lebens den Wirkungsort wechselten.

So kam Johann Otmar 1498 aus der freien Reichsstadt Reutlingen nach Tübingen, zog aber 1502 bereits nach Augsburg weiter, wo der Buchdruck lukrativer erschien. Etwas länger blieb der badische Drucker Thomas Anshelm, dessen Berufsweg von Straßburg über Pforzheim nach Tübingen und schließlich Hagenau (Elsass) führte. Während seiner Tübinger Jahre 1511-1516 erschienen einige Neuauflagen bereits in den früheren Wirkungsorten publizierter Werke. Der Ortswechsel ging nicht einher mit einer grundsätzlichen Änderung der gedruckten Literatur. Vielmehr wurden die Werke humanistischer Gelehrter der Region über den Wechsel an Druckern und Druckorten hinweg immer wieder neu gedruckt. Das galt z.B. für den Dichter und Tübinger Poesie-Professor Heinrich Bebel (1472-1518), dessen Lyrik und Briefe von Otmar 1501 (Fr.D.qt.262) und von Anshelm in Pforzheim (1508: z.B. Phil.qt.20; 1510: z.B. Phil. qt.22) und Tübingen (1511: Phil.qt.23) gedruckt wurden. Anshelm arbeitete in einem Netzwerk von Humanisten des südwestdeutschen Raums. Dazu zählte der zeitweise in württembergischen Diensten stehende Johannes Reuchlin (1455-1522), dessen Werke Anshelm auch in seiner Tübinger Zeit druckte. Charakteristisch ist die 1512 von Anshelm gedruckte Edition der lateinischen Lyrik des italienischen Renaissance-Philosophen Giovanni Pico della Mirandola (1470-1533) durch Jakob Spiegel (1483 bis ca. 1547) aus dem elsässischen Schlettstadt (Fr.D.qt.486). Die humanistischen Publikationen waren fast durchweg in lateinischer Sprache gehalten und verfolgten in positiv gehaltener Dikti-

1) Aleander, Hieronymus: Die Depeschen des Nuntius Aleander vom Wormser Reichstage 1521, übersetzt und erläutert von Paul Kalkoff. Schriften des Vereins für Reformationsgeschichte 17. Halle/Saale 1886, S. 44. 
on den Rekurs auf Sprache, Literatur und Philosophie der Antike. Apologetisch-polemische Werke wie die Streitschriften Reuchlins gegen Johannes Pfefferkorn und dessen antijüdische Agitation waren die Ausnahme. ${ }^{2}$ Philipp Melanchthon arbeitete von 1514 bis 1516 als Korrektor für Anshelm und verantwortete Neuauflagen verbreiteter humanistischer Werke. ${ }^{3}$ Das philologische Interesse verband Anshelm mit Melanchthon auch nach Zuwendung des letzteren zur Reformation. Anshelm druckte später in Hagenau einzelne Werke Melanchthons. ${ }^{4}$ Obwohl Anshelm der einzige in Württemberg tätige Drucker seiner Zeit war, wirkte sich nur in geringem Umfang die fachliche Breite der universitären Wissenschaft auf seine Produktion aus. ${ }^{5}$ Den Bericht Herzog Ulrichs über die Unruhen des Armen Konrad 1514 druckte Anshelm ohne ausdrückliche Nennung seines Namens. ${ }^{6}$ Die sich ab 1515 verschärfende Rücksichtslosigkeit in der Machtpolitik Herzog Ulrichs führte 1519 zu dessen Absetzung und könnte Anshelm 1516 dazu bewogen haben, in das sichere Hagenau zu ziehen. In der ersten Hälfte des 16. Jahrhunderts wurden in Stuttgart nur drei Jahre lang Bücher gedruckt und zwar durch Hans Werlich von Erfurt. Das politische Umfeld Württembergs ließ zunächst keine dauerhafte Etablierung eines Druckers zu. So wirkte Hans Werlich von Erfurt ab 1518 in Augsburg, von 1521 bis 1522 in Worms, von 1522 bis 1524 in Stuttgart, um dann die verbleibenden Jahre bis 1532 in Reutlingen zu drucken. Nach einer Übergangszeit kam es 1522 zur Herrschaft der Habsburger über Württemberg. Für die neuen Oberherren war es wichtig, offizielle Verlautbarungen und Berichte schriftlich fassbar zu machen und zu vervielfältigen. So konnte man Hans Werlich von Erfurt auch mit dem Angebot eines Hauses zur Einrichtung der Druckerei gewinnen.7 Staatliche Aufträge wie der Druck des Loblieds über den

2) Z.B. Reuchlin, Johannes: Defensio ... contra calumniatores suos Colonienses. Tübingen 1514 (HB 2208).

3) Z.B. Dialogus Mythologicus Bartolomaei Coloniensis, hrsg. von Philipp Melanchthon. Tübingen 1515 (HBF 1570).

4) Z.B. Melanchthon, Philipp: Institutiones rhetoricae. Hagenau 1521 (HB 42). 5) Z.B. die "Tabulae Astronomicae" des Tübinger Mathematikers Johannes Stöffler, die Anshelm 1514 druckte (Ra 16 Stoe 1).

6) Ulrich (Württemberg, Herzog): Warhafftig underrichtung der uffrürn unnd handlungen sich im fürstenthumb Wirtemperg begeben. Tübingen 1514 (HBF 4444).

7) Vgl. Reske, Christoph: Die Buchdrucker des 16. und 17. Jahrhunderts im deutschen Sprachgebiet. Auf der Grundlage des gleichnamigen Werkes von Josef Benzing. Wiesbaden 2007, S. 910.

8) Triumph vnd Victoria. Wie ... Ferdinandus: Printz und Infant in Hispanien, Ertzhertzog zu Osterreich ... auff den XXV. Tag des Monats May 1522 zu Stutgarten ingeritten unnd loblich empfangen ist. Stuttgart 1522 (R 16 Tri 1).

9) Der Lanndschafft zu Wirttemperg Freyhait. Gegeben durch Kaiser Karl V. und Ferdinand zu Stutgart am 28. May 1522. Stuttgart 1522 (HBFa 811).

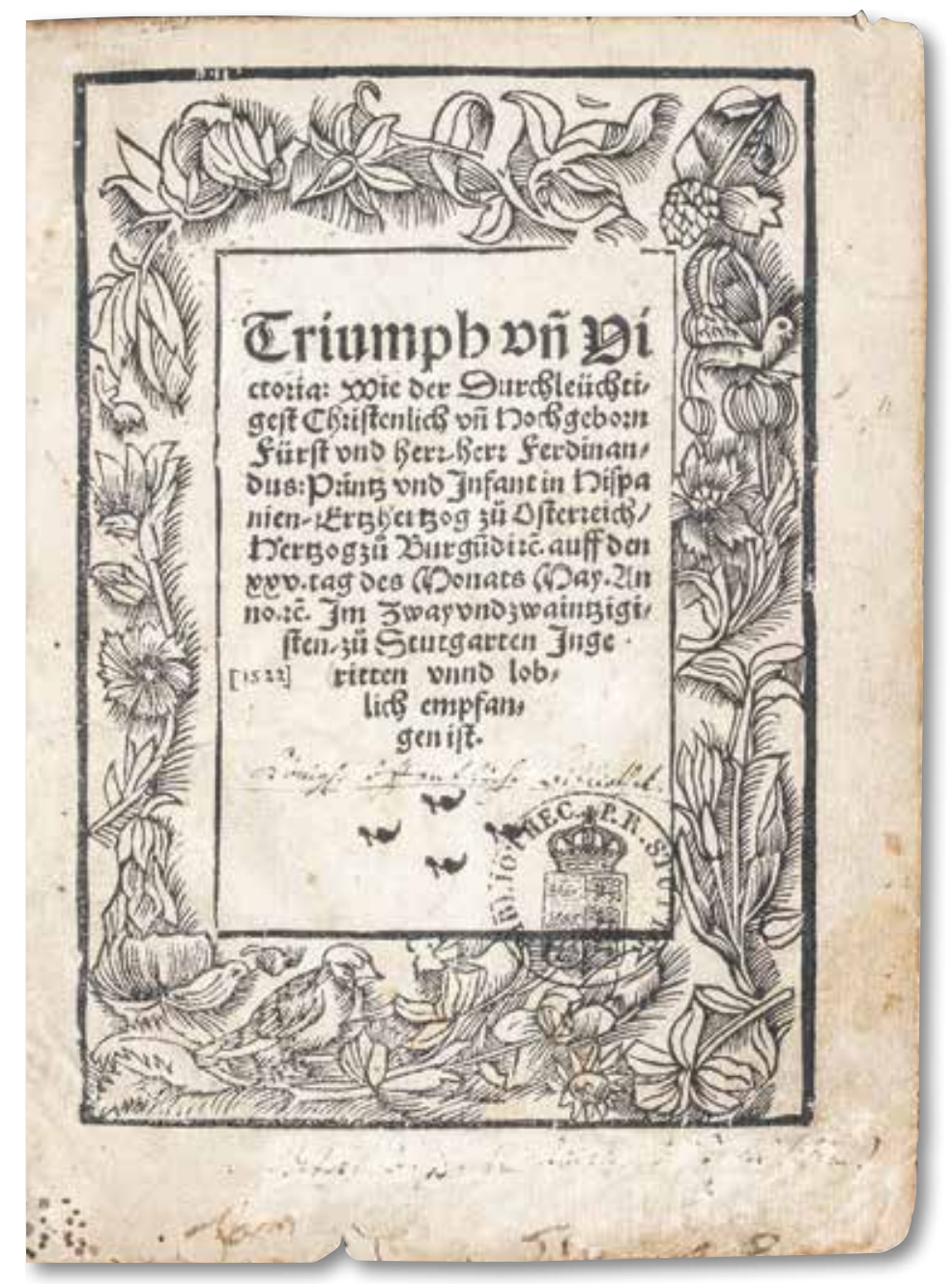

Abb. 2: Amtliche Schrift (R 16 Tri 1)

offiziellen Einzug des österreichischen Erzherzogs und späteren Kaisers Ferdinand I. im Jahr 1522 in Stuttgart waren charakteristisch. ${ }^{8}$

Ähnliches gilt für das kaiserliche Privileg zur Sicherung der Rechte der württembergischen Landschaft als Vertretung der führenden Kräfte in der kooperativen Verwaltung des Territoriums von 1522. ${ }^{9}$ Dieses Dokument druckte Werlich mit Summarien und breitem Rand für Notizen.

Die offiziellen Aufträge reichten jedoch nicht für das finanzielle Auskommen des Druckers. Mit großer Nachfrage auf dem Buchmarkt konnte man rechnen, wenn man kontroverstheologische Schriften aus Sicht der Reformation druckte. Hans Werlich folgte aus kommerziellen Erwägungen heraus diesem Trend, druckte die reformatorischen Schriften jedoch ohne Angaben zum Druckort und Drucker. Der Abgleich von Typographie und Holzschnittverzierungen mit den firmierten Drucken erlaubt jedoch die Zuordnung der Drucke zu Wer- 


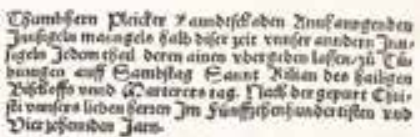

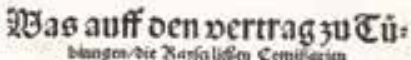

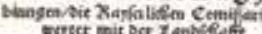

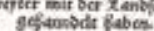

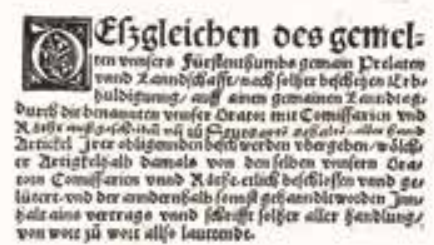

2) Jie Xaiferlicber JBaieftat

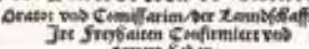

(0) 3r:B3arimilian won Jer

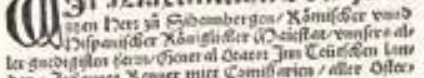
ben

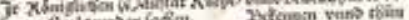
natsongaw

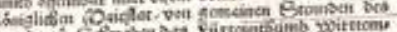

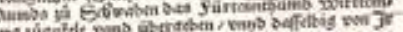

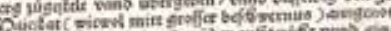

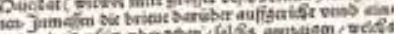

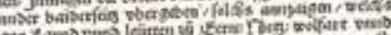

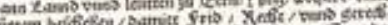

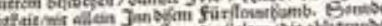

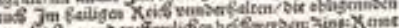

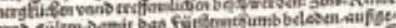

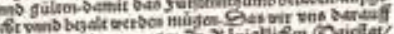

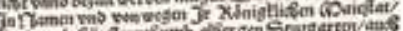

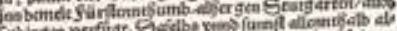

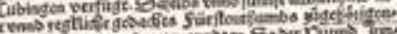

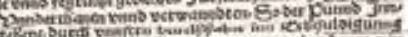

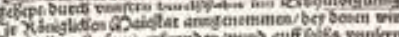

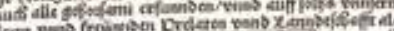

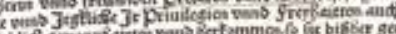

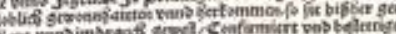

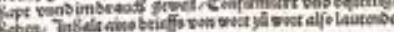

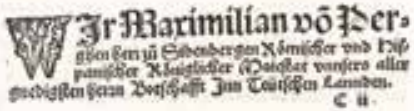

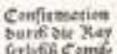
fortioficome arisu.

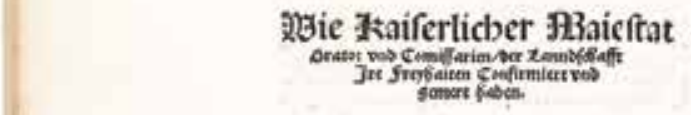

lich. Als Beispiele sind Nachdrucke von Luthers "Sermon von dem unrechten Mammon" (1522) sowie der deutschen Übersetzung seiner Antwort auf die antireformatorische Schrift König Heinrichs VIII. von England (1522) zu nennen. ${ }^{10}$ Einen hohen Aktualitätsgrad versprach die Kontroverse um den Widerruf reformatorisch orientierter Thesen durch den Magister Arsacius Seehofer († 1543) vor der Universität Ingolstadt am 7. September 1523 und die Antwort der Argula von Grumbach (1492-1554) darauf. ${ }^{11}$ Der Regionalbezug eines Autors erhöhte die Absatzchancen im Umland des Druckers. Deswegen druckte Werlich 1523 eine Verteidigungsschrift Luthers aus der Feder des ehemaligen Ulmer Franziskaners Heinrich von Kettenbach († ca. 1524). ${ }^{12}$ Erst in der Ausgabe einer reformationskritischen Schrift des Johannes Cochlaeus (1479-1552) deutete Werlich 1524 im Kolophon die Zuordnung zum Druckort an. ${ }^{13}$ Das geschah aber zurückhaltend, um in reformatorisch orientierten Kreisen keine negativen Assoziationen mit der Stuttgarter Offizin zu wecken. Die habsburgische
Herrschaft ließ es geraten erscheinen, reformatorische Schriften möglichst ohne Hinweise auf den Drucker herauszubringen. Die Bereitschaft, gegenreformatorische Schriften zu drucken, wurde aber vermutlich zu spät erkennbar, um Werlich vor Schwierigkeiten zu bewahren. Deswegen zog er nach Reutlingen weiter, wo er weitere reformatorische Werke druckte.

10) Luther, Martin: Ain Sermon von dem vnrechten Mammon. Luce am XVI. Stuttgart 1522 (R 16 Lut 11); Luther, Martin: Anntwurt deütsch Mar. Luthers auff Könnig Heinrichs von Engeland Buch. Stuttgart 1522 (R 16 Lut 12).

11) Die Artickel so Mayster Arsacius Sehofer von Münnchen durch die hohen Schul zu Jngelstat beredt, am Abent vnser Frawen Geburt nechstuer Erschinen widerrufft vnnd verworffen hat. Wie ain christliche Fraw des Adels in Bayern durch iren in götlicher Schrift wolgegründte Setbrieff die hohen Schul zu Ingelstat umb denselbigen Arsacius zu widersprechung des Wort Gotes betragt haten straffet. Stuttgart 1523 (R 16 Art 3).

12) Kettenbach, Heinrich von: Ein new Apologia unnd Verantworttung Martini Luthers wyder der Papisten Mortgeschray. Stuttgart 1523 (R 16 Ket 1).

13) Cochlaeus, Johannes: Responsio in epistolam cuiusdam Lutherani, Stuttgart 1524 (VD16 C 4376) (nicht in WLB). Textende und Kolophon: "Vale. Ex Tridento. III. Cal. Martij. Anno. M.D.XXIIIJ. // Stuttgardie. Sub serenissimo Principe, Ferdinando. Idibus lunij". VD16 = Verzeichnis der im deutschen Sprachraum erschienenen Drucke des 16. Jahrhunderts (http://www.vd16.de). 


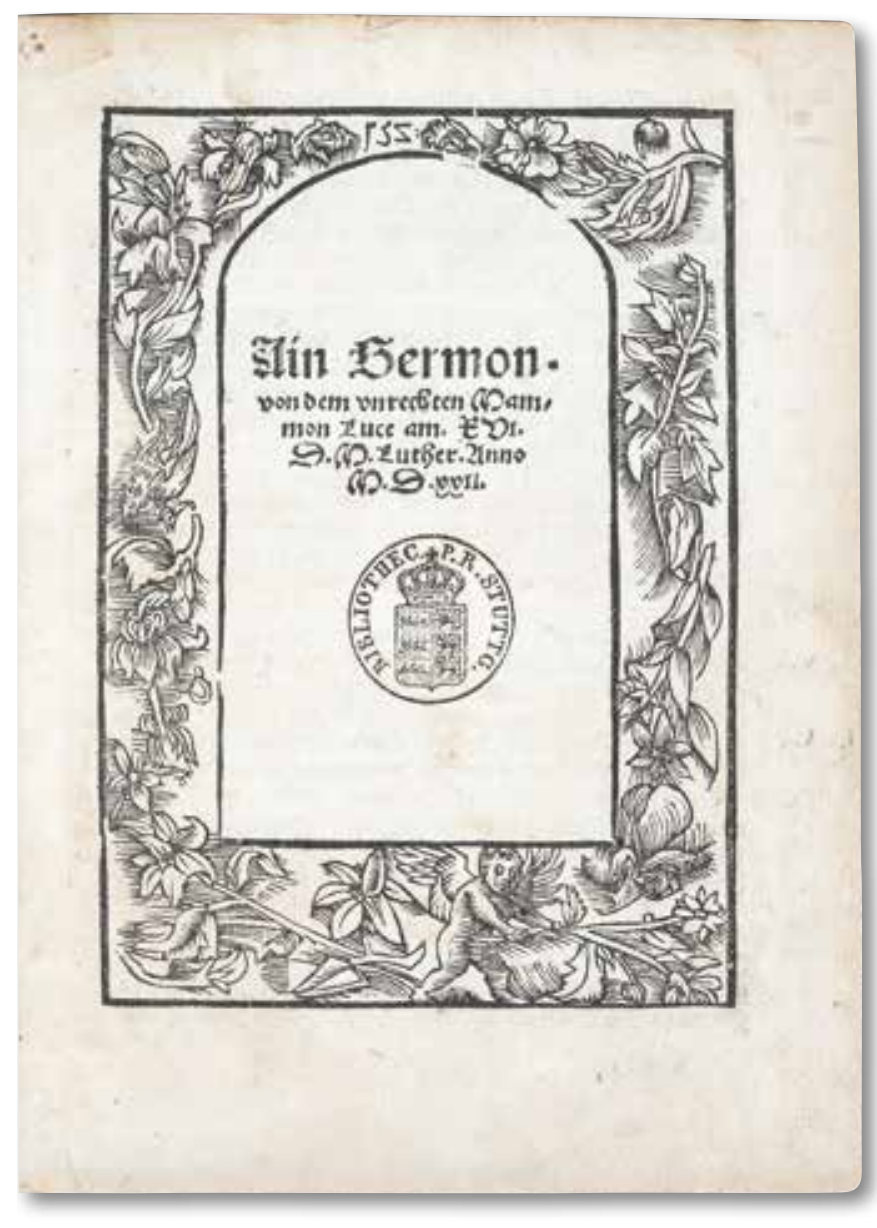

Abb. 4: Stuttgarter Ausgabe einer Luther-Flugschrift (R 16 Lut 11)

Dass Luthers Sendbrief an die Stadt Esslingen nicht in Esslingen, sondern als Erstausgabe bei Werlich 1523 in Stuttgart gedruckt wurde, zeigt, mit welchen finanziellen Risiken die Investitionen zur Unterhaltung einer Offizin einhergingen. ${ }^{14}$ In Esslingen siedelten sich nach dem Aufschwung des Buchdrucks in der Inkunabelzeit unter Konrad Fyner (bis 1479) erst im 17. Jahrhundert wieder Buchdrucker an. So übernahmen die Drucker des Territorialstaats Württemberg auch den Druck von Schriften mit Bezug auf die südwestdeutschen Reichsstädte.

Die prolutherischen Schriften des Esslinger Augustiners Michael Stifel (ca. 1487-1567) wurden 1522/1523 in Straßburg und Augsburg gedruckt. In den freien Reichsstädten des Südwestens konnten

14) Luther, Martin: Meynen liebenn Herrn und Freünden, in Christo, allen christlichen Bürgern zu Eßlinngen. Stuttgart 1523 (R 16 Lut 10). Nachdrucke in Straßburg, Nürnberg, Augsburg.

15) Blarer, Ambrosius: Christenlicher Abschid. Ulm: Hans Grüner, 1532 (Theol.qt.K.59). Die spiritualistischen Werke druckte Hans Varnier d.Ä. zwischen 1534 und 1562.

16) Z.B. Cochlaeus, Johannes: Ein christliche Vermanung der heyligen Stat Rom an das Teütschland, yr Tochter im christlichen Glauben. Tübingen: Morhart, 1524 (VD16 C 4363). Cochlaeus, Johannes: Sieben Kopffe Martini Luthers vom hochwirdigen Sacrament des Altars. Tübingen: Morhart, 1532 (VD16 C 4393). Dietenberger, Johann: Wider das unchristlich Buch Mart. Luth. von dem Missbrauch der Mess. Tübingen: Morhart, 1526 (VD16 D 1506).

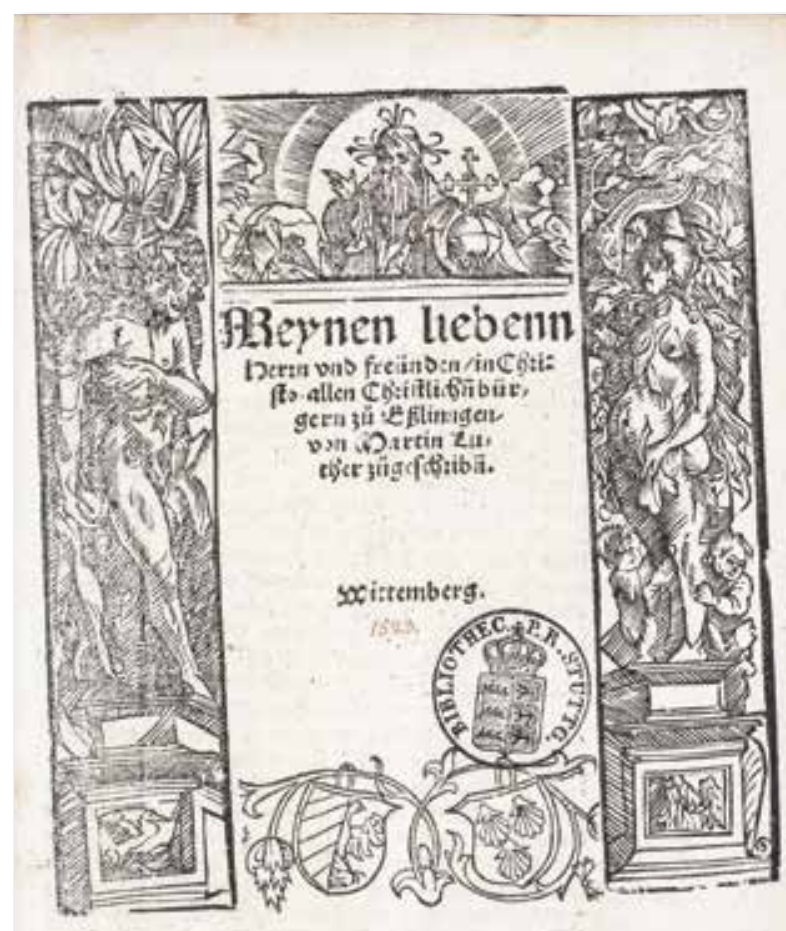

Abb. 5: Inhaltlicher Bezug zu Esslingen (R 16 Lut 10)

zwar früher als in Württemberg Pfarrer einzelner Gemeinden offen im Sinne der Reformation predigen. Jedoch blieb die konfessionelle Situation bis zum Augsburger Reichstag 1530 nicht eindeutig. Danach traf man, sofern überhaupt in den südwestdeutschen Reichsstädten gedruckt wurde, unter den Druckschriften auf die Vielfalt der einander widerstreitenden Strömungen der Reformation. Dies galt insbesondere für Ulm, wo neben einer Schrift Ambrosius Blarers (1492-1564) mit seiner zwischen Luther und Zwingli vermittelnden Haltung viele Werke des spiritualistischen Flügels der Reformation (Sebastian Franck, Kaspar Schwenckfeld) gedruckt wurden. ${ }^{15}$ Die konfessionspolitischen Vorgaben der Obrigkeit waren in Württemberg präziser als in den Reichsstädten und wurden im weiteren Verlauf des Jahrhunderts mit Auswirkungen auf den Buchdruck immer strenger gehandhabt.

Das ist auch daran erkennbar, dass insbesondere deutschsprachige Tübinger Drucke mit reformationskritischer Ausrichtung nicht in den Bestand der späteren Stuttgarter Konsistorialbibliothek bzw. Hofbibliothek gelangten, von wo sie in die WLB gekommen wären. ${ }^{16}$ Man wollte anders als 
bei der späteren Pflichtexemplarabgabe nicht die Buchproduktion des Landes dokumentieren und kontrollieren, sondern die Rechtgläubigkeit im lutherischen Sinne gewährleisten und abweichende Literatur makulieren. Gerade deutschsprachige Drucke galten als gefährlich, und seit der Reformation publizierten auch deren Gegner weitaus mehr volkssprachliche Schriften als zuvor.

Das Beispiel des Johannes Cochlaeus steht für die signifikanten Veränderungen der Buchproduktion, die mit der Reformation einhergingen. Bis zum Erscheinungsjahr 1520 sind im VD16 von inm 27 philologische und philosophische Werke - ausschließlich in lateinischer Sprache - nachgewiesen. ${ }^{17}$ Das Auftreten Luthers motivierte ihn zu einer thematischen Neuorientierung. Nun arbeitete er weit überwiegend an theologischen Themen.

Zudem schrieb er jetzt weniger sachlich-distanziert als polemisch. Die Reformation beflügelte auch bei ihren Gegnern die Produktivität erheblich und kam dem Buchdruck zugute. Für die Zeit von 1521 bis 1540 sind von Cochlaeus 188 Ausgaben im VD16 dokumentiert, davon $86(45,7 \%)$ in deutscher Sprache. Bei Ulrich Morhart dem Älteren in Tübingen wurden davon sechs gedruckt, darunter zwei deutsche Ausgaben. Das universitäre Umfeld und das noch vorhandene humanistische Netzwerk führten dazu, dass der Anteil der lateinischen Drucke gerade der papsttreuen Autoren in Tübingen weit über dem Durchschnitt im deutschen Sprachgebiet blieb. ${ }^{18}$ Die methodisch der humanistischen Praxis folgenden Neueditionen frühchristlicher oder scholastischer Autoren durch Cochlaeus in der Zeit nach 1520 verfolgten im Zeitkontext eher kontroverstheologische Ziele. ${ }^{19}$

Reichsweit sind hingegen im VD16 für 1521 bis 1540 derzeit 3.131 Ausgaben von Lutherschriften nachgewiesen. Davon wurden bei Morhart in Tübingen neun gedruckt - acht in deutscher Sprache und beschränkt auf die Jahre 1524 bis 1526. Tübingen gehörte aufgrund der österreichischen Herrschaft in Württemberg bis 1534 zu den wenigen Druckorten, in denen katholische Literatur gedruckt wurde. Dass hier trotz der staatlichen Restriktionen einige reformatorische Schriften herauskamen, erklärt sich aus der asymmetrischen Situation der Nachfrage. Man kann dies am quantitativen Verhältnis der Ausgaben ablesen. Die reformatorische Seite warf in weitaus höheren Anteilen deutsche Drucke auf den Markt. Dies beruht auf der publizistischen Strategie, die theologischen Diskussionen von den Universitäten in die Breite der Gesellschaft zu tragen. ${ }^{20}$ Auch erforderte der für Luther existenzielle Zusammenhang von Wort Gottes und Glaube das sprachlich-intellektuelle Verstehen der Glaubensinhalte durch die Laien.

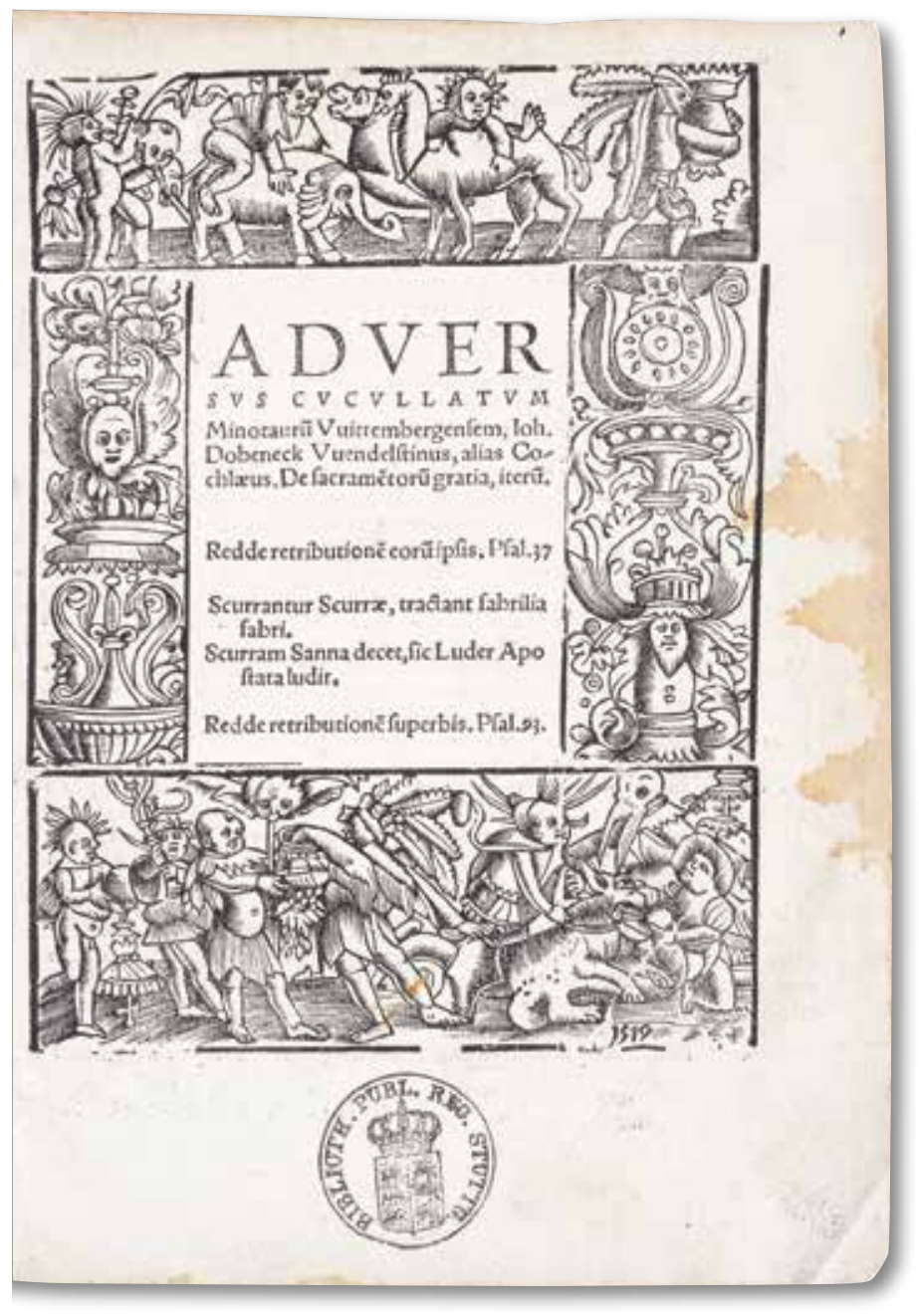

Abb. 6: Reformationskritisches Werk (R $16 \operatorname{Coc} 1)$

Ulrich Morhart dem Älteren (ca. 1490-1554) aus Augsburg gelang es, dauerhaft eine Offizin an einem württembergischen Ort zu etablieren. Dabei zählte er anfänglich auch zu den wandernden Druckern und druckte zunächst ab 1519 in Straßburg und erst ab 1523 in Tübingen. Er begründete eine Druckerdynastie. Nach seinem Tod führte zunächst

17) Zuletzt eine lateinische Grammatik, Straßburg 1519 (HB 532).

18) Vgl. Kaspar Schatzgeyer: 35 Ausgaben im Zeitraum 1521 bis 1540, davon 16 (45,7\%) deutsch; bei Morhart in Tübingen 10 Ausgaben, ausschließlich in Latein. Johann Eck: 108 Ausgaben im Zeitraum 1521 bis 1540, davon 41 (38\%) deutsch; bei Morhart in Tübingen 4 Ausgaben, davon 1 deutsch.

19) Cochlaeus, Johannes (Hrsg.): Opuscula Maxentii lohannis, Servi Dei, Theologi Antiqui. Köln 1526 (Theol.oct.11755). Cochlaeus, Johannes (Hrsg.): Rvperti Abbatis Tvitiensis De Victoria Verbi Die. Köln 1529 (HBb 1943).

20) Für den Zeitraum 1521 bis 1540 sind im SWB 2.760 Luther-Ausgaben nachgewiesen, davon 2.033 in deutscher Sprache $(73,4 \%)$. 
seine Witwe Magdalena mit ihren Söhnen die Druckerei weiter, bis letztere unter ihrem Familiennamen Gruppenbach firmierten. Möglich wurde dies vor allem durch das geschickte Agieren Morharts und die sich stabilisierenden politischen Rahmenbedingungen in Württemberg. Morhart wusste, dass von den Habsburgern reformationskritische Aktivitäten unterstützt wurden und es wegen der katholisch bleibenden Fürsten und Bevölkerungsteile in Deutschland einen gewissen Marktanteil für papstfreundliche Literatur gab. Außer in Tübingen wurden in der frühen Phase der Reformation vor allem in Köln, Leipzig und Dresden katholische Werke in Deutschland gedruckt. Für 1523 bis 1534 sind von Morhart 105 Drucke im VD16 dokumentiert. Davon sind 64 (61\%) theologischen Themen gewidmet. 47 Schriften lassen sich durch Autoren und Inhalte eindeutig dem Spektrum katholischer Kontroversliteratur zuordnen (44,8\% der Gesamtproduktion, 73,4\% der theologischen Titel). Hierzu gehören viele bereits in der Titelformulierung ausgesprochen polemisch gehaltene Schriften. ${ }^{21}$ Immerhin 17 reformatorisch orientierte bzw. von mit der Reformation verbundenen Autoren verfasste Werke druckte Morhart bis 1534. Allerdings handelt es sich häufig um solche Schriften, die für die katholischen Theologen und österreichischen Oberherren weniger anstößig waren. Das gilt etwa für Luthers Stellungnahmen gegen die aufständischen Bauern (1525) und für Kommentare zu konfessionell wenig umstrittenen Teilen der Bibel (z.B. Petrusbriefe). ${ }^{22}$ Oder Morhart druckte eine Schrift, die neben die Position Luthers die Gegenposition stellt, etwa Luthers Briefwechsel mit Herzog Georg von Sachsen (1526). ${ }^{23}$ Alle Luther-Drucke erschienen vorsichtshalber ohne Hinweis auf den Drucker. Morhart wollte am einträglichen Druck reformatorischer Schriften partizipieren, ohne sich in seinem Umfeld in Misskredit zu bringen. Er

21) Z.B. Cochlaeus, Johannes: Adversus cucullatum Minotaurum Vuittembergensem. Tübingen 1523 ( $R 16$ Coc 1) [= "Gegen den verkappten Minotaurus aus Wittenberg"].

22) Luther, Martin: Wider die mordischen unnd reübischen Rotten der Bawren. Tübingen 1525 (Theol.qt.K.739); Luther, Martin: Ermanunge zum frid, auff die zwoelff Artickel der Bawrschafft in Schwaben. Tübingen 1525 (Theol.qt.K.502). Kommentare zu Petrusbriefen 1524 (VD16 L 4597 bzw. L 4609).

23) Ein Sendbrieff doctor Martini Luthers ann Hertzog Georg zu Sachssen ... darinn er jn freüntlich ermant zu dem wort Gottes zu dretten. Ein antwort Hertzog Georg zu Sachssen ... an Doctor Martinum. Tübingen 1526 (VD16 L 5923).

24) Lambertus Hersfeldensis: Qvisqvis Es Gloriae Germanicae \& maiorum studiosus, hoc utare ceu magistro libello. Tübingen 1525 (VD16 ZV 23175: mit Vorrede) (HBF 1438). Version ohne Vorrede: VD16 L161.

25) Z.B. Melanchthon, Philipp: Annotationes in Evangelium Matthaei. Tübingen 1523 (Theol.oct.11989).

26) Cochlaeus, Johannes: De libero arbitrio hominis, adversvs locos communes Philippi Melanchthonis. Tübingen 1525 (Theol.oct.3280).

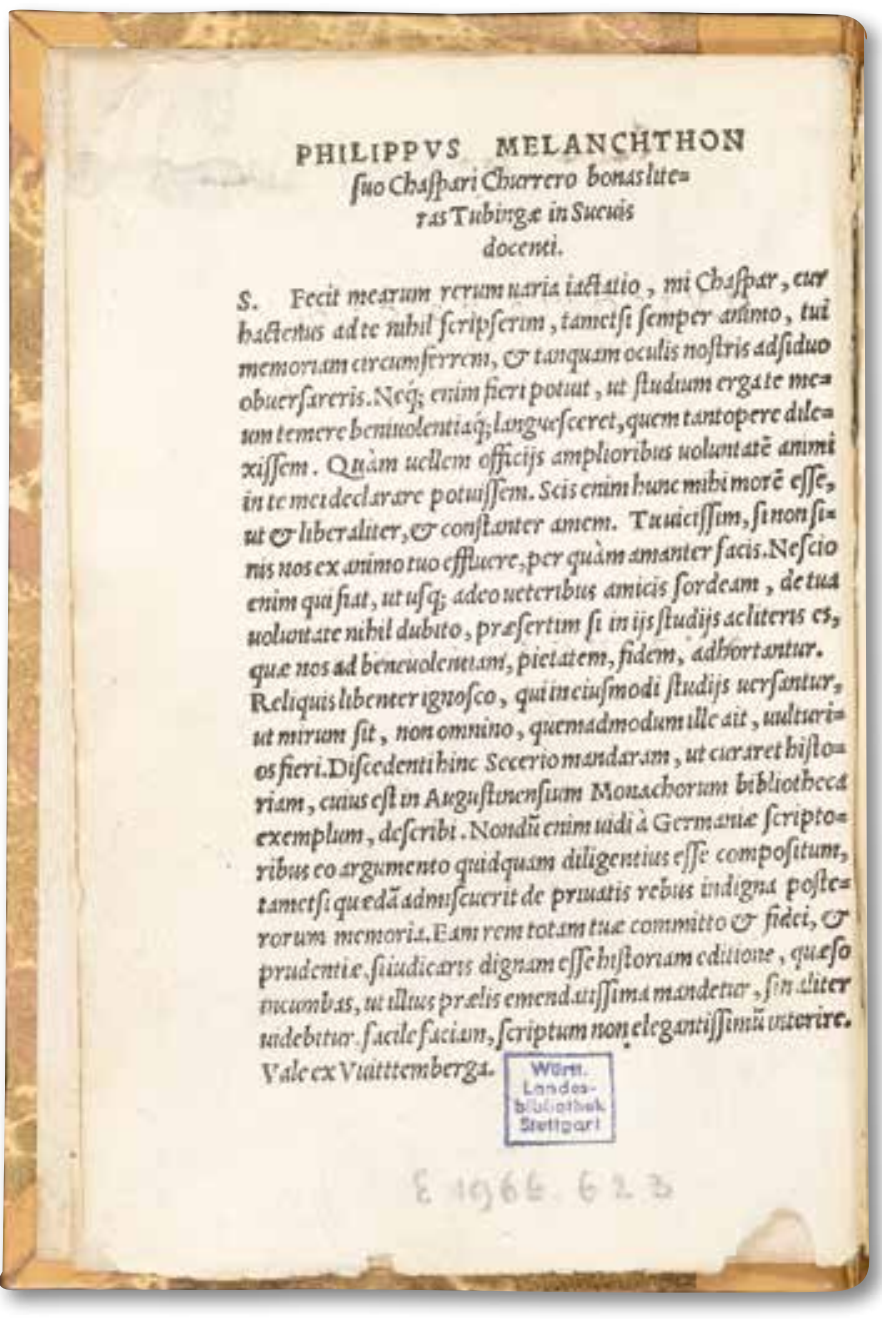

Abb. 7: Druck mit Vorrede Melanchthons (HBF 1438)

hatte beide konfessionellen Bevölkerungsteile im Blick und passte sein Angebot entsprechend an. So brachte er die theologisch unverdächtige deutsche Chronik des Lambert von Hersfeld in zwei Fassungen heraus, nämlich mit und ohne Vorrede Melanchthons. ${ }^{24}$

Melanchthons philologische Expertise wurde von den Gegnern der Reformation weiterhin geschätzt und ermöglichte die Publikation exegetischer Kommentare und editorischer Arbeiten, an denen er beteiligt war. ${ }^{25}$ Gerade das Beispiel Melanchthons zeigt aber, wie tief die Spaltung der bestehenden humanistischen Netzwerke durch die Kontroverse um die Reformation reichte. Im engeren Sinne konfessionelle Werke Melanchthons wie die "Loci communes" oder die "Confessio Augustana" konnten von Morhart nicht gedruckt werden, wohl aber Gegenschriften dazu. ${ }^{26}$

Die Edition des griechischen Neuen Testaments durch Erasmus von Rotterdam wurde in humanis- 
tisch orientierten Kreisen hoch anerkannt. Schwieriger war es mit der Einschätzung der darauf basierenden lateinischen Übersetzung. Diese war einerseits philologisch exakter als die Vulgata, stand andererseits in Konkurrenz zu dieser kirchenamtlich verbindlich eingeführten Bibel. Morhart brachte 1523 eine Ausgabe des lateinischen Neuen Testaments in der Übersetzung des Erasmus heraus, die außerdem Einführungen Melanchthons zum Römerbrief und 1. Korintherbrief enthielt. Dies deutet mehr als die vagen Ausführungen Morharts in seiner Vorrede an den Leser die tatsächlichen Sympathien des Druckers für die Reformation an. ${ }^{27}$

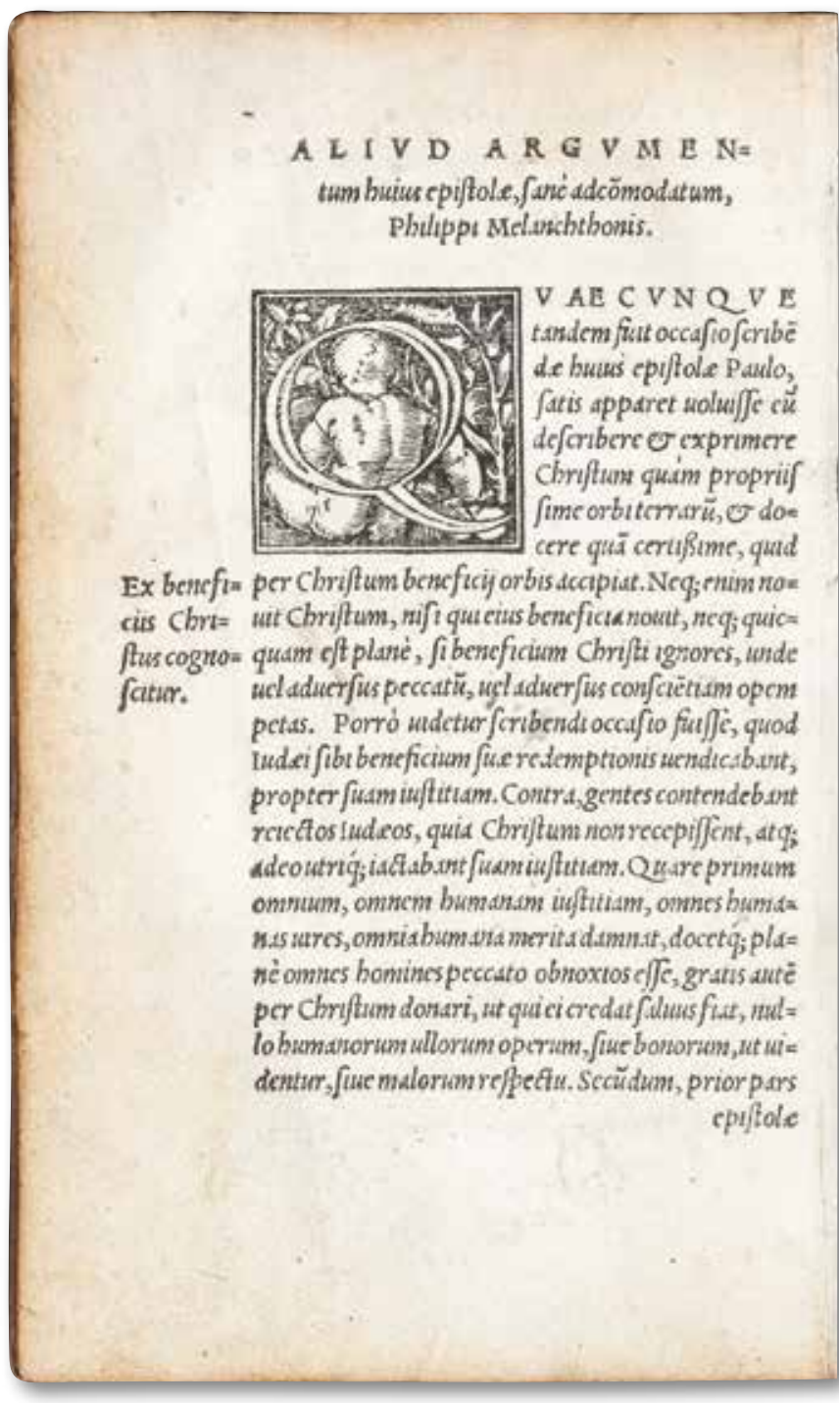

Abb. 8: Einführung Melanchthons zum Römerbrief (B lat.1523 07)

Andererseits wollte sich Morhart nicht die Absatzmöglichkeiten einer deutschen Bibelausgabe katholischer Übersetzer und Kommentatoren entgehen lassen. So druckte er 1532 im Auftrag der Kölner Offizin Peter Quentels eine Ausgabe des deutschen Neuen Testaments Hieronymus Emsers mit Korrekturen von Johann Dietenberger. ${ }^{28}$ Immerhin sollte die nächste deutsche Bibelausgabe in Württemberg erst 1590/1591 gedruckt werden.

\section{Morhart konnte editorische Großprojekte wie} Bibeln auch deswegen finanzieren, weil er als einziger Drucker in Württemberg auch amtliche Schriften im Auftrag staatlicher Stellen druckte. ${ }^{29}$ Hinzu kamen Lehrbücher im universitären Zusammenhang bzw. für den Schulbedarf. Die Hochschätzung der Reformatoren für die Volkssprache neben den antiken Sprachen wirkte sich hier positiv so aus, dass nicht nur ein lateinisches, sondern auch ein deutsches Rhetorik-Lehrbuch gedruckt wurde. Unter dem Titel "Rethorica unnd Formularium Teütsch" erschien das Werk Alexander Huges aus Calw ab 1528 in mehreren Auflagen bei Morhart. ${ }^{30}$ Die philologischen Werke humanistischer Autoren traten in der öffentlichen Wahrnehmung seit der Reformation zwar zurück. Einige Autoren garantierten aber über alle politischen und konfessionellen Umbrüche hinweg ein gewisses Maß an Kontinuität und verlässlichen Absatzmöglichkeiten. Das ließe sich etwa von den regional verankerten Autoren Johannes Stöffler und Johannes Reuchlin sagen. ${ }^{31}$

Das umsichtige und eher auf Ausgleich bedachte Agieren Morharts, andererseits die Notwendigkeit, amtliche Schriften im eigenen Land drucken zu müssen, ließ die Tübinger Offizin auch über die Rückkehr Herzog Ulrichs an die Macht im Jahr 1534 hinaus bestehen. Von 1535 bis 1554 erschienen laut VD16 weitere 154 Drucke Morharts. Die theologischen Werke waren nun durchweg reformatorisch, im engeren Sinne zunehmend lutherisch orientiert. Daneben wurden konfessionell neutrale wissenschaftliche bzw. amtliche Schriften gedruckt. Gerade die kirchliche und staatliche Neuorganisation durch Herzog Ulrich bedurfte der publizistischen Bekanntgabe und Verbreitung. Das gilt vor allem für die Kirchenordnung (R 16 Kir 1), die

27) Erasmus, Desiderius (Übers.): Novi Testamenti totius aeditio longe optima \& accuratißima. Tübingen 1523 (B lat.1523 07). Evtl. Anspielung auf das reformatorische "Allein die Gnade" in Morharts Vorrede, Bl. Aa $1 \mathrm{~V}$ : "à cuius misericordia omnis salus nostra pendet" (= "von seiner [Gottes; C.H.] Barmherzigkeit hängt all unser Heil ab").

28) Das gantz neü testament. Tübingen 1532 (Bb deutsch 1532 03).

29) Z.B. Karl V. (Heiliges Römisches Reich, Kaiser): Keiserliche Freiheit, wie die jungsten der Juden auch ächter halb auff werenden Reichßtage zu Augspurg erlangt ist. Tübingen 1530 (HBFa 528).

30) Erstausgabe 1528 (HBb 627). Folgeauflagen 1530, 1535, 1537, 1540.

31) Stöffler wurde bereits 1514 von Anshelm in Tübingen gedruckt (Ra 16 Stoe 1), dann von Morhart ab 1523. Die "Ephemerides" erschienen bei Morhart 1531, 1533, 1548, 1549. Morhart druckte eine ReuchlinSchrift 1529 (Theol.qt.5656). 


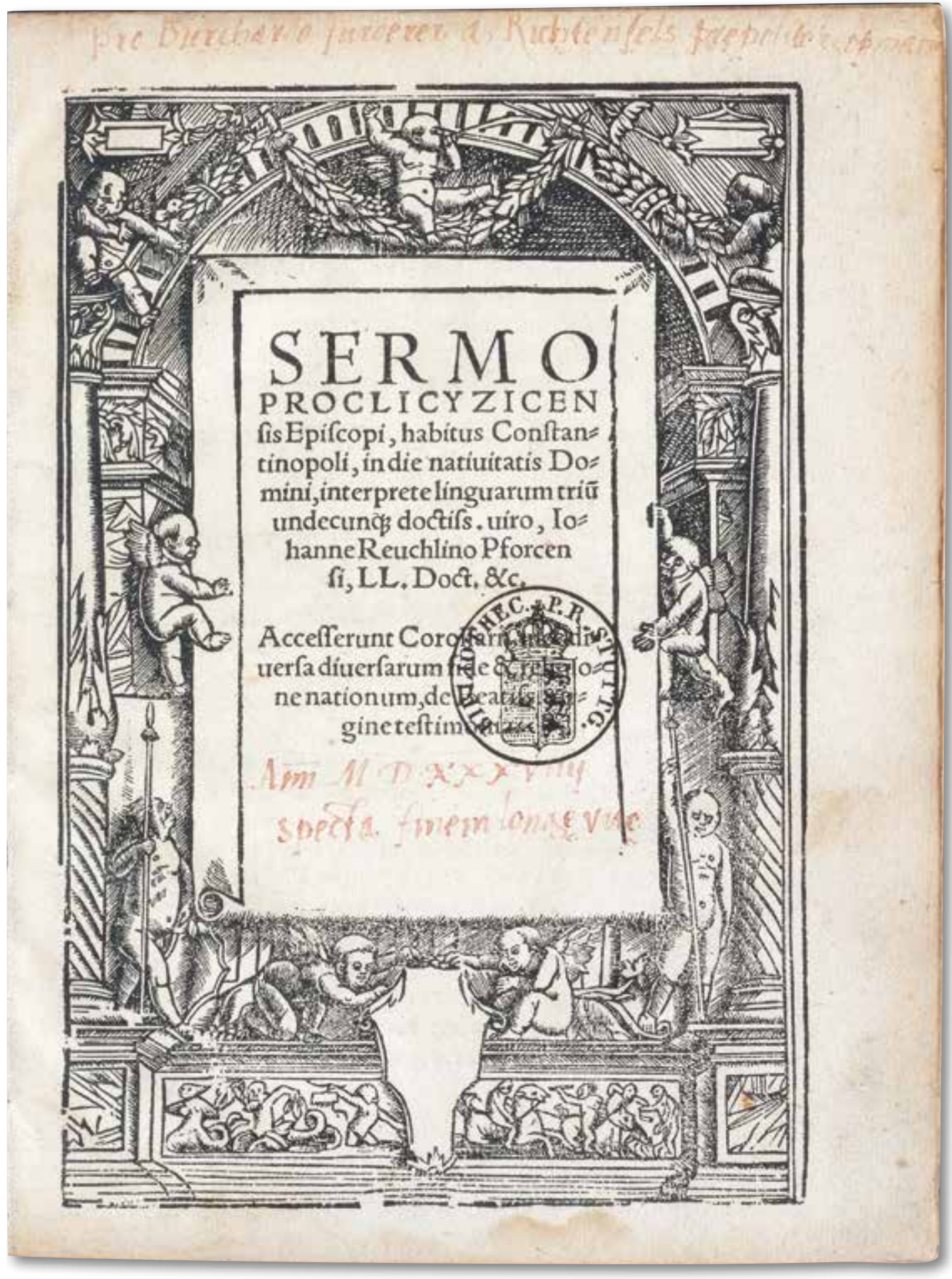

Abb. 9: Reuchlin-Druck bei Morhart (Theol.qt.5656)

Landesordnung (Wirt.R.qt.104), die Eheordnung (HBF 2756) und die Einrichtung eines Gemeinen Kastens zur Armenfürsorge (HBF 2759) (alle 1536).

Wegen der vorsichtigen Haltung Morharts hatten sich andere, vor allem süddeutsche Druckorte für Werke reformatorisch orientierter Theologen des Südwestens etabliert. So wurden Schriften von Johannes Brenz (1499-1570) ab 1523 gedruckt, von Morhart in Tübingen aber erst ab 1552, dann mit steigender

Tendenz. Mit der Etablierung der Reformation ging allerdings die Produktion apologetischer und kontroverstheologischer Schriften zunächst drastisch zurück. Unter den ab 1535 gedruckten Schriften Morharts befanden sich nur noch $26 \%$ aus dem Bereich der Theologie, überwiegend von regional bedeutsamen Theologen wie Ambrosius Blarer, Philipp Melanchthon, Kaspar Gräter, Erhard Schnepf und Primož Trubar. Morhart konnte an die vorreformatorische Drucktradition in der Weise anknüpfen, dass der Anteil profaner, wissenschaftlicher Literatur im Umfeld der Universität anstieg. Erst mit dem Regierungsantritt Herzog Christophs und dessen gegenüber Ulrich ausgeprägterem Interesse an Theologie wurde die Offizin Morhart wieder stärker zu einer Druckerei theologischer Schriften. Der dezidiert lutherische Charakter des württembergischen Kirchenwesens bildete sich von der zweiten Hälfte des 16. Jahrhunderts an auch mit Hilfe des Buchdrucks heraus.

Christian Herrmann 